\title{
Une situation de " post-front pionnier » : le cas de la région rurale de Ciriaco (ouest Maranhão - Brésil)
}

\section{Stéphanie Nasuti}

\section{(2) OpenEdition}

Journals

Édition électronique

URL : http://journals.openedition.org/cdg/2182

DOI : $10.4000 /$ cdg. 2182

ISSN : 2107-7266

Éditeur

UMR 245 - CESSMA

\section{Référence électronique}

Stéphanie Nasuti, «Une situation de « post-front pionnier » : le cas de la région rurale de Ciriaco (ouest Maranhão - Brésil) », Carnets de géographes [En ligne], 1 | 2010, mis en ligne le 01 octobre 2010, consulté le 24 septembre 2020. URL : http://journals.openedition.org/cdg/2182 ; DOI : https://doi.org/ $10.4000 /$ cdg. 2182

Ce document a été généré automatiquement le 24 septembre 2020.

\section{cc) $(1)$}

La revue Carnets de géographes est mise à disposition selon les termes de la Licence Creative Commons Attribution - Pas d'Utilisation Commerciale - Pas de Modification 4.0 International. 


\title{
Une situation de « post-front pionnier » : le cas de la région rurale de Ciriaco (ouest Maranhão - Brésil)
}

\author{
Stéphanie Nasuti
}

1 Cette étude présente les composantes spatio-temporelles ayant structuré une région d'arrière- front pionnier de la frange orientale de l'Amazonie brésilienne (pré-Amazonie maranhense, Imperatriz), dont les paysages sont progressivement dominés par la forêt de babaçu, forêt anthropique par excellence. Cinquante ans après les débuts de l'occupation, on passe d'un "Maranhão caboclo ", qui envisageait son avenir autour de la noix du palmier babaçu (Orbignya spp.), à un « Maranhão moderne » qui, bien que conservant un certain poids du "traditionalisme", s'oriente, avec sa sidérurgie, vers de nouveaux modes de production.

2 Nous avons ainsi cherché à expliciter les étapes qui sous-tendent la construction d'un territoire, le premier front pionnier de l'Amazonie, à partir d'un raisonnement spatiotemporel, que nous avons poursuivi en multipliant les échelles géographiques et temporelles. Dans un premier temps, afin de saisir les conditions d'organisation du peuplement, nous revenons sur les trajectoires migratoires de la première génération ayant afflué massivement dans les années soixante, que nous comparons aux actuelles stratégies des jeunes générations.

3 Les itinéraires migratoires, obtenus par le biais de la méthodologie d'enquête biographique Ebimaz ${ }^{1}$, ont permis de réaliser une cartographie inédite et donc de préciser quelques-uns des flux démographiques caractéristiques des situations d'arrière-front.

4 Les parcours migratoires de la première génération de migrants, qui se caractérisaient par des trajets au long cours, sont remplacés par d'autres formes de mobilité spatiale, de plus courte portée, que l'on qualifie de « circulation » car elles se caractérisent par des stratégies d'aller- retour autour d'un point d'ancrage, souvent construites dans une complémentarité de résidence entre ville et campagne. La problématique des migrations des jeunes touche directement au développement des zones rurales, car elle 
soulève les questions à la fois de la disponibilité foncière, de l'emploi local de jeunes de mieux en mieux éduqués ainsi que de l'évolution agricole, et va jusqu'à poser la question du développement durable.

5 Cependant, la possibilité de stabilisation des familles sur leur lieu de résidence dépend pour beaucoup des conditions foncières. Dans cette région, l'évolution foncière, inhérente à la consolidation d'un front pionnier, s'est singularisée par diverses interventions directes de l'Etat Fédéral, privilégiant tour à tour la régularisation individuelle pour rétablir la paix sociale (GETAT) puis l'usufruit collectif des terres pour préserver l'environnement (aires protégées). Nous avons ainsi reconstitué les étapes de la chaîne foncière jusqu'à l'implantation de la réserve extractiviste de Ciriaco, première aire protégée dédiée au babaçu et cas d'étude central de ce travail. Centrés sur cet exemple, nous avons cherché à montrer dans quelles conditions, dans une région au cadastre longtemps inexistant, des petits agriculteurs ont pu accéder durablement à la propriété. Pour cela, des sources variées ont été mobilisées et présentées sous forme de schémas et de cartes: en plus des registres des cabinets notariaux et du matériel de l'INCRA ${ }^{2}$, nous avons eu accès à des archives inédites datant de l'époque de la dictature militaire, matériel de première main grâce auquel les conditions d'installation de l'époque ont pu être reconstituées.

6 Cette réserve extractiviste, de faible dimension ( $8084 \mathrm{ha}$ ), n'est qu'un point dans la frange pionnière amazonienne, où pourtant elle figure toujours en bonne place par sa notoriété institutionnelle environnementale. Sa présence, incongrue dans le panorama régional, a le mérite d'introduire la problématique du développement durable dans cette zone d'arrière-front et d'y discuter notamment la viabilité de la gestion partagée d'une ressource naturelle dans un cadre foncier collectif. D'autre part, l'implantation de ce dispositif amène la question de la modernisation des produits de l'extractivisme et de la capacité des populations rurales à s'insérer dans les circuits commerciaux. La transformation de la noix de babaçu, activité en désuétude, exprime pourtant une identité collective forte, qui s'incarne dans un mouvement social emblématique des transformations du milieu politico-associatif rural, qui entre dans une démarche réflexive et acquiert plus d'autonomie, concourant à structurer les communautés rurales en zone de front pionnier.

7 Cette dynamique régionale se complète par les problématiques actuelles du secteur sidérurgique, activité pionnière de fort impact, mais dont l'implantation dans les années quatre-vingts a donné une envergure nationale à la région d'Imperatriz. Aujourd'hui, pour assurer la "durabilité » de ses activités et garantir sa place sur le marché du lingot de fer- gueuse, le secteur est contraint de déployer un véritable système spatial articulé autour de la plantation forestière (eucalyptus) et de sa transformation en charbon végétal.

Il s'agit donc bien de la question de l'avenir de cette région devenue, en une quarantaine d'années, un "post-front pionnier ", à tel point que son rattachement à l'Amazonie légale est fortement remis en question. C'est ainsi qu'au cours de cette réflexion la notion de "post- front pionnier" s'est imposée à nous; même si l'expression plus spatiale « d'arrière-front » a été dans le texte largement utilisée, celle de "post-front pionnier ", incluant davantage la dimension temporelle, nous semble mieux adaptée pour exprimer la réalité des transformations de ce grand ouest maranhense. 
Fiche informative

\section{Discipline \\ Géographie}

\section{Directeur}

Martine DROULERS, IHEAL-Université Paris III

\section{Université}

Institut des Hautes Études de l'Amérique latine / Paris 3

Membres du jury de thèse, soutenue le 9 juin 2010

- Alfredo W. Berno de ALMEIDA, Anthropologue, Professeur à l'Universidade

Estadual do Amazonas, Rapporteur

- Martine DROULERS, Géographe, Directrice de recherches au CNRS, Directrice de thèse

- Afrânio GARCIA, Anthropologue, Maître de Conférences (HDR) à l'EHESS, Président

- Pernette GRANDJEAN, Géographe, Professeur à l'Université de Reims, Rapporteur

- François-Michel LE TOURNEAU, Géographe, Chargé de recherche (HDR)au CNRS,

Examinateur

\section{Situation professionnelle actuelle}

Chargée de mission pour l'Institut des Amériques

\section{Contact de l'auteur}

steph.nasuti[at]gmail.com

\section{NOTES}

1. Développée dans le cadre du programme de recherche Duramaz (ANR blanc, 2006-2009), coordonné par Martine Droulers et François-Michel Le Tourneau, qui visait à analyser en détail les déterminants géographiques, démographiques et socio- économiques de treize expériences de développement durable menées en Amazonie brésilienne.

2. Institut National de Colonisation et Réforme Agraire.

INDEX

Thèmes : Carnets de soutenances 